OPEN ACCESS

Edited by:

Maurizio Acampa,

Siena University Hospital, Italy

Reviewed by:

Marialuisa Zedde

Local Health Authority of Reggio

Emilia, Italy

Raffaele Ornello,

University of L'Aquila, Italy

*Correspondence:

Bo Xu

bxu@citmd.com

Jin-qing Yuan

dr_jinqingyuan@sina.com

Specialty section: This article was submitted to

Stroke,

a section of the journal

Frontiers in Neurology

Received: 12 July 2021 Accepted: 29 September 2021 Published: 02 November 2021

Citation:

$X u$ J-j, Jia S-d, Zhu P, Jiang L, Jiang $P$,

Song $Y$, Zhao $X-y, L i J-X$, Chen J,

Yang Y-j, Gao R-I, Qiao S-b, Xu B and

Yuan J-q (2021) Does Prior Stroke

Predict Long-Term Recurrent Stroke

After Percutaneous Coronary Intervention? Five-Year Results From a Large Cohort Study.

Front. Neurol. 12:740136. doi: 10.3389/fneur.2021.740136

\section{Does Prior Stroke Predict Long-Term Recurrent Stroke After Percutaneous Coronary Intervention? Five-Year Results From a Large Cohort Study}

\author{
Jing-jing Xu, Si-da Jia, Pei Zhu, Lin Jiang, Ping Jiang, Ying Song, Xue-yan Zhao, \\ Jian-xin Li, Jue Chen, Yue-jin Yang, Run-lin Gao, Shu-bin Qiao, Bo Xu* and Jin-qing Yuan* \\ Department of Cardiology, Fuwai Hospital and Cardiovascular Institute, National Center for Cardiovascular Diseases, Chinese
Academy of Medical Sciences and Peking Union Medical College, Beijing, China
}

Background: We found a positive correlation between the prior stroke history and recurrent stroke in patients who underwent percutaneous coronary intervention $(\mathrm{PCl})$ in our previous study, which indicated the close interaction of stroke and cardiovascular diseases. However, it is unclear whether prior stroke is still associated with worse prognosis at a longer follow-up period.

Methods: A total of 10,724 coronary heart disease (CHD) patients who received $\mathrm{PCl}$ from January to December 2013 were prospectively enrolled and were subsequently divided into the prior stroke $(n=1,150)$ and non-prior stroke $(n=9,574)$ groups according to their history. Baseline characteristics and 5-year outcomes were recorded.

Results: Patients with prior stroke had more clinical risk factors, as well as more extensive coronary artery lesions. Although in-hospital outcomes were similar between patients from the two groups, the 5-year follow-up result revealed that patients with prior stroke experienced higher incidence of stroke, major adverse cardiac and cerebrovascular events (MACCEs), all-cause death, and cardiac death (7.0 vs. 3.0\%, $p<0.001 ; 25.9$ vs. $20.3 \%, p<0.001 ; 5.3$ vs. $3.5 \%, p=0.002 ; 3.1$ vs. $2.1 \%$, $p=0.032$, respectively). After the propensity score matching, the 5 -year stroke rate was still higher in the prior stroke group (6.8 vs. 3.4\%, $p=0.001$ ). The multivariable regression analysis also identified the prior stroke as a risk predictor of the 5-year stroke $(\mathrm{HR}=2.011,95 \% \mathrm{Cl}: 1.322-3.059, p=0.001)$.

Conclusions: Coronary heart disease patients with prior stroke who received $\mathrm{PCl}$ had a higher incidence of 5-year long-term adverse cardiovascular and cerebrovascular events, especially recurrent stroke. Prior stroke was a strong risk predictor of future stroke events.

Keywords: prior stroke, coronary heart disease, $\mathrm{PCl}-$ percutaneous coronary intervention, predictor, long-term outcome 


\section{INTRODUCTION}

The incidence of cerebrovascular diseases, especially stroke, has been increasing in low- and middle-income countries. Stroke is the leading cause of death and disability globally (1). Individuals with atherosclerosis have an increased risk of stroke (2). About $19 \%$ of stroke occurs simultaneously with cardiac events, and this, combined with cerebrovascular disease, may lead to worse prognosis for coronary heart disease (CHD) patients (3). As an essential means of revascularization, percutaneous coronary intervention (PCI) can improve therapeutic outcomes and the quality of life (4). However, the risk of recurrent cardiocerebrovascular events increases in PCI patients with prior stroke, which complicates the decision on the treatment strategy choice $(5,6)$. As pointed out by Doehner et al. from the European Society of Cardiology Council on Stroke, the stroke care is an interdisciplinary challenge, and the close collaboration of stroke physicians and cardiologists is essential.

Our previous study reported that in CHD patients with prior stroke WHO received PCI, the incidence of 2-year major adverse cardiac and cerebrovascular event (MACCE), all-cause death, and other adverse events was higher than those without prior stroke, especially recurrent stroke, which remained at significantly higher risk after the confounder adjustment (7). However, with the extension of the period, whether the prior stroke is still related to the worse prognosis is not very unclear. Therefore, we further investigated the in-hospital outcomes and conducted a 5-year long-term follow-up to evaluate the exact impact of prior stroke on the prognosis of CHD patients after PCI, and we added subgroup analyses with high risk of clinical adverse events including older age, premature CHD (PCHD), and acute coronary syndrome (ACS) to assist the choice of clinical treatment regimens.

\section{METHODS}

\section{Study Population}

A total of 10,724 consecutive CHD patients who received PCI from January to December 2013 were enrolled in this singlecenter study (Fuwai Hospital, National Center for Cardiovascular Diseases, Beijing, China). Patients were divided into prior stroke group $(n=1,150)$ and non-prior stroke group $(n=9,574)$. All patients were provided with written informed consent before the intervention, and the study has been approved by the Ethics Committee of the Hospital (ethical application no. IRB2012-BG006, approval no. 2013-449).

\section{Procedural and Medications}

The strategy, stent, and drugs used during PCI were at the physician's discretion. If there was no contraindication of taking long-term aspirin or P2Y12 inhibitors, selective patients received oral aspirin (loading dose $300 \mathrm{mg}$ ) and clopidogrel (loading dose $300 \mathrm{mg}$ ) or ticagrelor (loading dose $180 \mathrm{mg}$ ) at least $24 \mathrm{~h}$ before the procedure. Acute coronary syndrome patients [ST segment elevation myocardial infarction (MI) or non-ST elevation-ACS] scheduled for PCI received the same dose of aspirin and ticagrelor or clopidogrel (loading dose 300 or $600 \mathrm{mg}$ ) as soon as possible.
Heparin of 1,000 IE was received at the beginning of the procedure; the 60 IE heparin/kg body weight was also conducted if the PCI has to be executed. Whether to use glycoprotein IIb/IIIa inhibitors was subject to operator's judgment. After the procedure, aspirin was prescribed at the dose of $100 \mathrm{mg}$ per day indefinitely; clopidogrel $75 \mathrm{mg}$ per day or ticagrelor $90 \mathrm{mg}$ twice per day was advised for at least 1 year after PCI.

\section{Patient Follow-Up}

All patients were evaluated by clinic visit or by phone at the first-month, sixth-month, first-year, second-year, and fifth-year time points after procedure. All adverse events were evaluated by a group of independent clinical physicians and were carefully checked and confirmed.

\section{Endpoints and Definitions}

The primary endpoint was defined as MACCEs, a composite of all cause death, MI, stent thrombosis, repeat revascularization, and stroke during the 5-year follow-up. Secondary endpoint consisted of cardiac death, bleeding, and components of the primary endpoint. Death that could not be attributed to a noncardiac etiology was considered as cardiac death. Myocardial infarction was defined by the third universal definition of MI (8). Revascularization was defined as the repeated revascularization for ischemic symptoms and events driven by PCI or surgery of any vessel. Unplanned target vessel revascularization (TVR) was defined as repeat percutaneous intervention or surgical bypass of any segment of the target vessel for ischemic symptoms and events driven (9). Stent thrombosis was defined according to the Academic Research Consortium definition, including definite, probable, and possible categories in the analysis (9). Bleeding was quantified according to the Bleeding Academic Research Consortium (BARC) definition criteria, including types 2, 3, and 5 in the analysis (10). The SYNTAX score is summarized from SYNTAX trial (Synergy between PCI with TAXUS drug-eluting stent and Cardiac Surgery) to compare the MACCE after coronary artery bypass grafting (CABG) or PCI. Scoring was performed by professional coronary intervention technicians, which was based on the qualitative and quantitative characterization of coronary artery disease by including 11 angiographic variables that take into consideration lesion location and characteristics. The treatment scheme was finally selected by the interventional operators (11).

\section{Statistical Analyses}

Continuous variables were compared by Student's $t$-test as appropriate, and categorical variables were analyzed using Pearson's chi-square test. Continuous variables were presented as mean \pm standard deviation, and categorical variables are presented as number with frequency. The 5-year survival curves were calculated using the Kaplan-Meier estimates and were tested using log-rank method for endpoints. Confounders were adjusted using the Cox proportional-hazards regression, where results were expressed as hazard ratios (HRs) with corresponding 95\% confidence intervals (CIs). All clinically and statistically significant covariates were entered into the model with adjustments in the multivariate analysis. Due to 
substantial differences between the groups, we further performed a propensity score analysis that matched the following patient characteristics: age, gender, hypertension, diabetes mellitus, hyperlipidemia, smoking, prior $\mathrm{MI}$, prior revascularization procedure, the SYNTAX score before PCI, and the residual SYNTAX score. Patients from the non-prior stroke group were

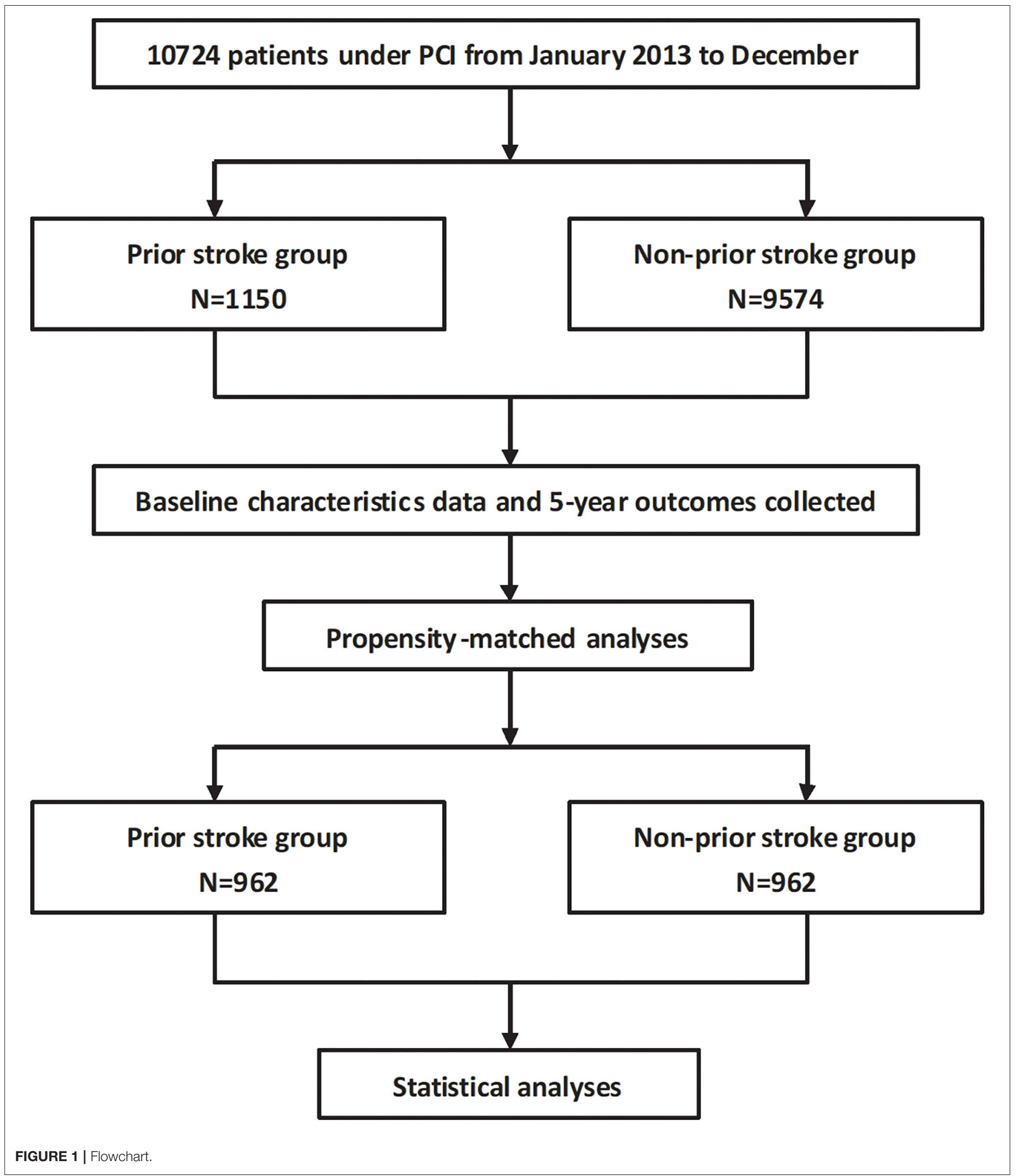


TABLE 1 | Baseline and propensity score matched data of two groups.

\begin{tabular}{|c|c|c|c|c|}
\hline \multicolumn{3}{|c|}{ Baseline } & \multicolumn{2}{|c|}{ After propensity score matched analyses } \\
\hline $\begin{array}{c}\text { Prior stroke } \\
\text { group } \\
n=1,150\end{array}$ & $\begin{array}{c}\text { Non-prior stroke } \\
\text { group } \\
n=9,574\end{array}$ & $P$-value & $\begin{array}{c}\text { Prior stroke } \\
\text { group } \\
n=962\end{array}$ & $\begin{array}{c}\text { Non-prior stroke } \\
\text { group } \\
n=962\end{array}$ \\
\hline
\end{tabular}

\section{Demographics \\ Age (years) \\ Female (\%) \\ BMl $\left(\mathrm{kg} / \mathrm{m}^{2}\right)$ \\ Risk factors \\ Hypertension (\%) \\ Diabetes mellitus (\%) \\ Hyperlipidemia (\%) \\ Smoker (\%) \\ Biochemical index \\ Hemoglobin ( $\mathrm{g} / \mathrm{L})$ \\ Platelet (*109/L) \\ HbAlc (\%) \\ TC (mmol/L) \\ LDL-C (mmol/L) \\ HDL-C (mmol/L) \\ $\mathrm{TG}$ (mmol/L) \\ eGFR}

Family history of CAD (\%)

White blood cell (*109/L)

Diseased coronary vessels

$\begin{array}{lc}\text { Syntax score before PCl } & 12.25 \pm 8.41 \\ \text { Residual SYNTAX score } & 4.19 \pm 6.27 \\ \text { LAD (\%) } & 1,025(89.1) \\ \text { LCX (\%) } & 227(19.7) \\ \text { RCA (\%) } & 220(19.1) \\ \text { Left main or three-vessel } & 50(4.4) \\ \text { disease (\%) } & \\ \text { Cardiovascular conditions } & \\ \text { ACS (\%) } & 694(60.3) \\ \text { Cardiac shock (\%) } & 7(0.6) \\ \text { Previous MI (\%) } & 251(21.8) \\ \text { Previous PCI (\%) } & 280(24.3) \\ \text { Previous CABG (\%) } & 31(2.7) \\ \text { LVEF (\%) } & 62.57 \pm 7.42\end{array}$

$278(24.2)$

$643(56.4)$

$6.92 \pm 1.94$

$6.74 \pm 1.26$

$4.19 \pm 1.05$

$2.45 \pm 0.89$

$1.03 \pm 0.28$

$1.69+0.95$

$80.05 \pm 26.26$

$63.12 \pm 8.90$
$325(28.3)$
$25.98 \pm 3.00$

$57.80 \pm 10.30$
$2,127(22.2)$
$25.93 \pm 3.20$

$\begin{array}{cc}<0.001^{\star} & 63.33 \pm 8.73 \\ <0.001^{\star} & 284(29.5) \\ 0.57 & 25.94 \pm 2.98\end{array}$

$5,965(62.3)$

\section{$2,807(29.3)$}

6,357 (64.4)

2,373 (24.8)

$138.10 \pm 16.02$

$202.06 \pm 54.04$

$5,480(58.7)$

$6.92 \pm 1.95$

$141.38 \pm 15.84$

$203.74 \pm 54.78$

$6.60 \pm 1.24$

$4.21 \pm 1.08$

$2.51 \pm 0.91$

$1.03 \pm 0.28$

$1.79 \pm 1.10$

$84.67 \pm 28.26$

$11.64 \pm 8.08$

$3.32 \pm 5.61$

8,677 (90.6)

$1,606(16.8)$

$1,689(17.6)$

407 (4.3)

$5,737(59.9)$
$23(0.2)$
$1,810(18.9)$
$2,360(24.7)$
$406(4.2)$
$62.77 \pm 7.35$

$<0.001^{*}$

$<0.001^{*}$

$<0.001^{*}$

0.334

0.015

0.948

0.328

$0.001^{*}$

$0.013^{*}$

$0.024^{\star}$

0.824

$0.004^{*}$

$<0.001^{\star}$

$0.016^{\star}$

$<0.001^{*}$

0.102

$0.012^{\star}$

0.212

0.872

0.781
$0.025^{\star}$
$0.010^{*}$
0.425
$0.008^{*}$
0.425
$<0.001^{*}$
$786(81.7)$

$367(38.1)$

$713(74.1)$

$237(24.6)$

$522(54.3)$

$6.71 \pm 1.70$

$138.48 \pm 15.69$

$199.27 \pm 51.08$

$6.72 \pm 1.19$

$4.10 \pm 1.04$

$2.41 \pm 0.87$

$1.04 \pm 0.28$

$1.72 \pm 0.99$

$79.89 \pm 26.62$

$11.77 \pm 8.01$

$4.02 \pm 6.03$

858 (89.2)

$184(19.1)$

$181(18.8)$

$42(4.4)$

$506(52.6)$
$0(0)$
$233(24.2)$
$231(23.7)$
$30(3.1)$
$3.546 \pm 6.7988$

$63.43 \pm 9.26$
$293(30.5)$
$25.83 \pm 3.16$

0.822

0.654

0.449

0.592

0.237

0.641

0.487

$\begin{array}{ll}224(23.3) & 0.487 \\ 515(53.5) & 0.087\end{array}$

$6.69 \pm 1.84 \quad 0.863$

$138.67 \pm 16.03 \quad 0.799$

$198.11 \pm 51.29 \quad 0.622$

$6.76 \pm 1.23 \quad 0.446$

$\begin{array}{ll}4.18 \pm 1.07 & 0.088\end{array}$

$\begin{array}{ll}2.47 \pm 0.88 & 0.147\end{array}$

$1.07 \pm 0.29 \quad 0.240$

$1.73 \pm 1.02 \quad 0.819$

$79.36 \pm 28.23 \quad 0.673$

$11.96 \pm 8.23 \quad 0.606$

$3.81 \pm 5.95 \quad 0.431$

860 (89.4) $\quad 0.883$

$175(18.2) \quad 0.598$

$178(18.5) \quad 0.861$

$45(4.6) \quad 0.809$

$\begin{array}{cc}526(54.7) & 0.361 \\ 0(0) & 1.000 \\ 226(23.5) & 0.708 \\ 228(24.0) & 0.873 \\ 26(2.7) & 0.587 \\ 63.414 \pm 7.5874 & 0.690\end{array}$

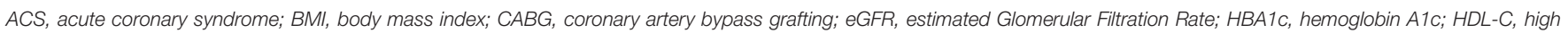

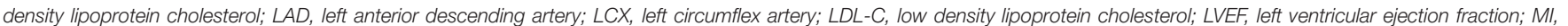

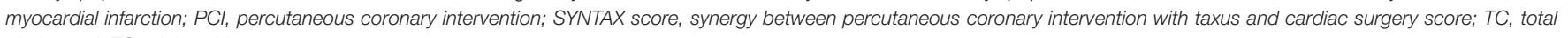
cholesterol; TG, triglyceride.

Data are expressed as mean \pm standard deviation; or counts (percentage).

${ }^{*} p<0.05$.

matched with those from the prior stroke group according to their propensity score, resulting in 962 pairs of propensitymatched patients ( $n=1,924$ in total) in whom long-term outcomes were determined. Older age ( $\geq 75$ years), PCHD (male $\leq 50$ years, female $\leq 60$ years), ACS, cardiac dysfunction [left ventricular ejection fraction (LVEF) $\leq 40 \%]$, hypertension, and diabetes mellitus were brought into subgroup analysis. The effect of the subgroup variables and prior stroke on the risk of long-term recurrent stroke was performed on Cox multivariate regression analysis. The results were expressed by the HR $(95 \% \mathrm{CI})$ and interaction $p$-value. All statistical analyses were performed at a significance level of two-sided $p$-value $=0.05$ with the software of SPSS version 19.0 (IBM Corporation, Armonk, New York). The flowchart is shown in Figure 1. 


\section{RESULTS}

\section{Baseline Characteristics}

A total of 10,724 CHD patients who have undergone PCI were enrolled in this study. The median follow-up time was 5.1 years (1,861 days) with a response rate of $91.5 \%$. Among these patients, $1,150(10.7 \%)$ had prior stroke, while 9,574 (89.3\%) did not.

Baseline and angiographic characteristics of the two groups are displayed in Table 1. Compared with the non-prior stroke group, patients with prior stroke were older, contained more female, and were more likely to have CHD risk factors, such as hypertension, diabetes mellitus, and hyperlipidemia. They also had more extensive coronary artery disease (higher SYNTAX score before PCI and residual SYNTAX score), more prior MI, and revascularization history (Table 1). After propensity score matching, all baseline variables were comparable between the two groups, as shown in Table 1.

\section{Outcomes}

Before matching, unadjusted in-hospital and 5-year clinical outcomes are shown in Table 2. Patients with or without prior stroke had similar incidence of in-hospital adverse cardiovascular and cerebrovascular events. The 5-year follow-up showed significantly higher rates of MACCE, all-cause death, cardiac death, and stroke ( 25.9 vs. $20.3 \%, p<0.001 ; 5.3$ vs. $3.5 \%, p$ $=0.002 ; 3.1$ vs. $2.1 \%, p=0.032 ; 7.0$ vs. $3.0 \%, p<0.001$, respectively) in the prior stroke group. After propensity score matching, however, there was no difference between two groups in the incidence of primary endpoint except for the 5-year stroke (6.8 vs. $3.4 \%, p=0.001$ ) (Table 2 ). The Kaplan-Meier estimates showed a similar trend (Figure 2).

The multivariable regression analysis identified prior stroke as a risk predictor of 5-year stroke before the propensity score matching ( $\mathrm{HR}=1.962,95 \% \mathrm{CI}: 1.520-2.532, p<0.001)$. Prior stroke remained a strong independent risk predictor of 5-year stroke after the propensity score matching $(\mathrm{HR}=2.011,95 \% \mathrm{CI}$ : 1.322-3.059, $p=0.001$ ) (Table 3).

\section{Subgroup Analysis}

The subgroup analyses with high risk of clinical adverse events included those with age $\geq 75$ years, PCHD, ACS, cardiac dysfunction, hypertension, and diabetes mellitus. They showed that ACS, hypertension, and diabetes mellitus had significant interaction with prior stroke history to increase the risk of 5-year stroke (HR $=1.579,95 \% \mathrm{CI}$ : 1.045-2.387, interaction $p=0.030 ; \mathrm{HR}=1.519,95 \%$ CI: 1.023-2.258, interaction $p=0.038 ; \mathrm{HR}=2.120$, 95\% CI: $1.390-3.234$, interaction $p<0.001$, respectively) (Figure 3).

\section{DISCUSSION}

This is a study focusing on the prognosis of CHD patients with or without prior stroke who underwent PCI in the largest cardiovascular center in China. The major findings of this study were as follows: (1) it was common that CHD patients undergoing PCI had a prior stroke history; (2) patients with

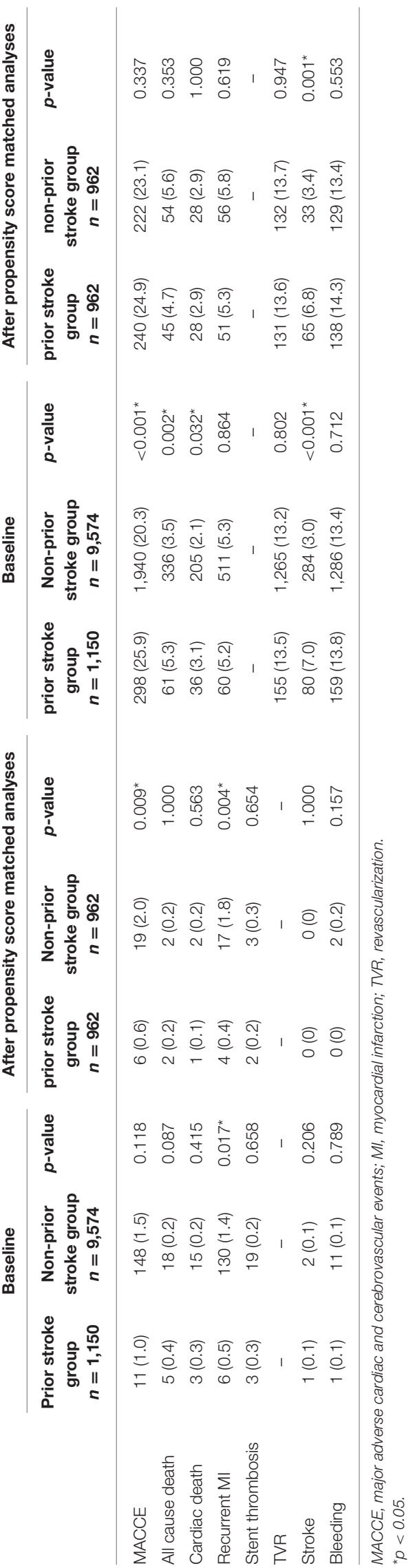




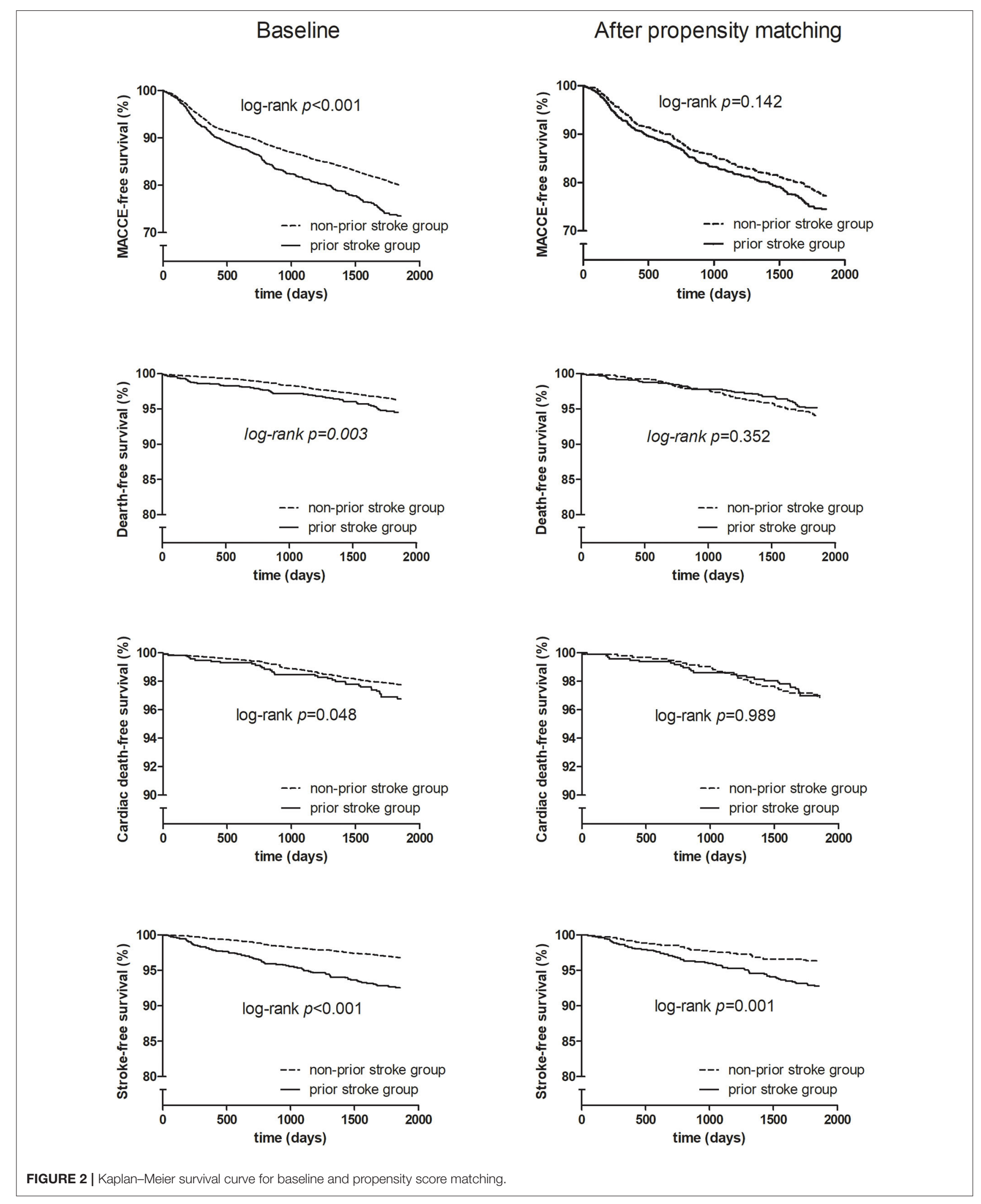


TABLE 3 | Multivariable regression analyses of prior stroke for 5-years long-term prognosis.

\begin{tabular}{|c|c|c|c|c|c|c|}
\hline & \multicolumn{3}{|c|}{ Baseline } & \multicolumn{3}{|c|}{ After propensity score matched analyses } \\
\hline & HR & $95 \% \mathrm{Cl}$ & $p$-value & HR & $95 \% \mathrm{Cl}$ & $p$-value \\
\hline MACCE & 1.200 & $1.059-1.360$ & $0.004^{\star}$ & 1.093 & $0.911-1.312$ & 0.339 \\
\hline All cause death & 1.119 & $0.848-1.477$ & 0.427 & 0.879 & $0.591-1.308$ & 0.525 \\
\hline Cardiac death & 1.079 & $0.753-1.547$ & 0.679 & 1.076 & $0.636-1.820$ & 0.785 \\
\hline Recurrent Ml & 0.919 & $0.700-1.207$ & 0.543 & 0.898 & $0.614-1.312$ & 0.578 \\
\hline TVR & 1.062 & $0.896-1.260$ & 0.489 & 0.994 & $0.780-1.266$ & 0.958 \\
\hline Stroke & 1.962 & $1.520-2.532$ & $<0.001^{\star}$ & 2.011 & 1.322-3.059 & $0.001^{*}$ \\
\hline Bleeding & 1.061 & $0.896-1.255$ & 0.493 & 1.080 & $0.849-1.374$ & 0.529 \\
\hline
\end{tabular}

MACCE, major adverse cardiac and cerebrovascular events; MI, myocardial infarction; TVR, revascularization. ${ }^{*} p<0.05$.

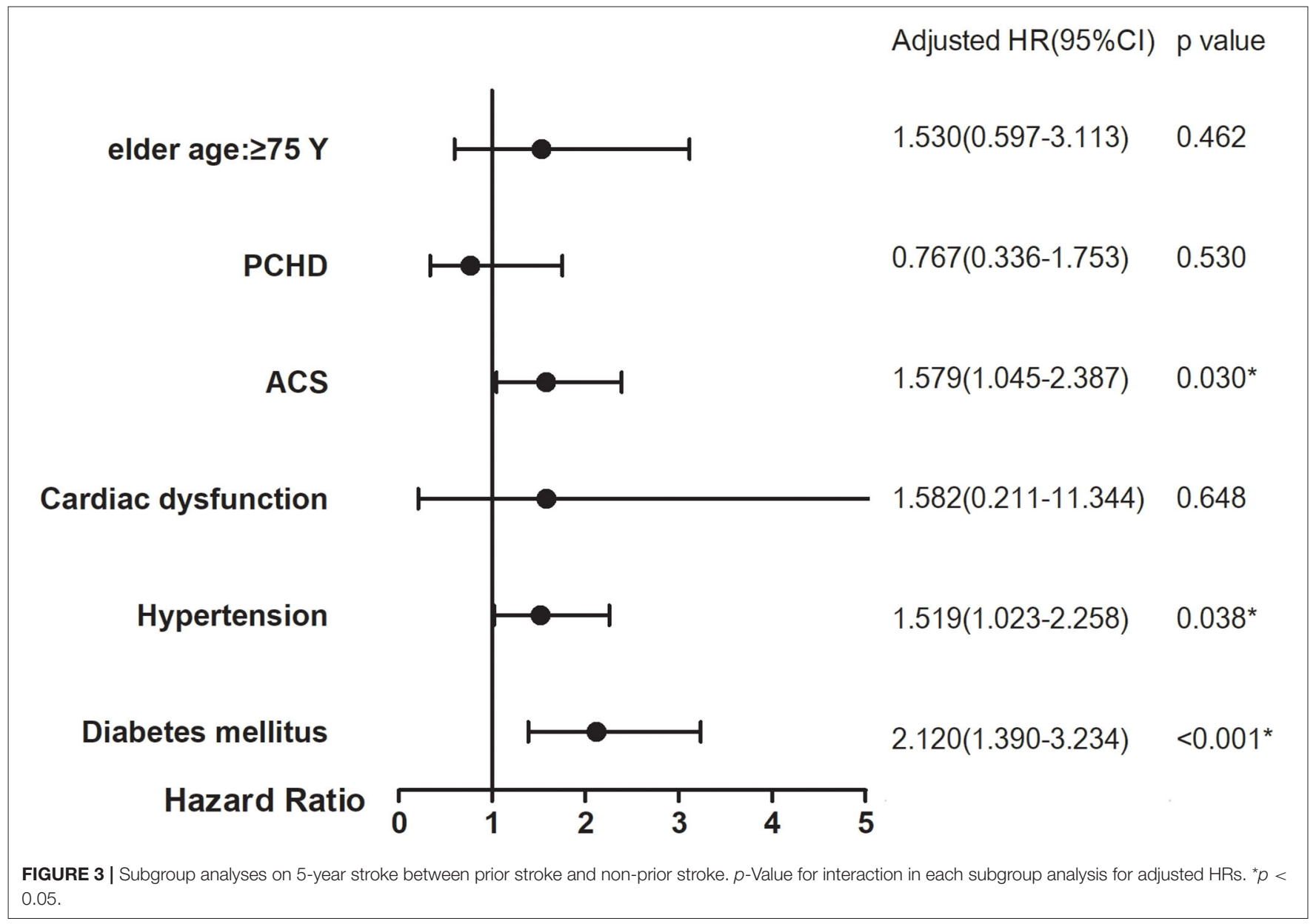

prior stroke had more high-risk baseline characteristics and were more likely to suffer severe coronary artery diseases; (3) although the incidence of in-hospital MACCE with or without prior stroke was similar, the 5-year follow-up showed a significantly higher incidence of recurrent stroke and MACCE in patients with prior stroke; (4) for CHD patients who underwent PCI, prior stroke was a strong risk predictor of future stroke events.

\section{Prevalence of Prior Stroke in Coronary Heart Disease Patients Undergoing Percutaneous Coronary Intervention}

Prevalence of stroke varies in nations around the globe, where in China the rate is reported to be $2.6-7.2$ per 1,000 persons (12). Stroke and CHD usually share many risk factors, and CHD patients are frequently complicated by stroke. Previous studies reported that the proportion of patients with $\mathrm{CHD}$ combined 
with prior stroke/transient ischemic attack (TIA) is about 13.3$19.7 \%$, which is related to race, age, and other factors $(13,14)$. In our cohort of CHD patients undergoing PCI in the largest cardiovascular center in China, we found that $10.7 \%$ of the patients had a history of prior stroke, which reflected the realworld situation of China.

\section{High-Risk Clinical Characteristics of Patients With Prior Stroke}

Patients with cardiovascular diseases and prior stroke were more likely to have more atherosclerotic risk factors, such as higher age, diabetes mellitus, hypertension, hyperlipidemia, chronic kidney disease, and peripheral arterial disease (15). Similarly, CHD patients with prior stroke in our study had worse baseline characteristics, including higher age, female gender, and more CHD risk factors such as hypertension, diabetes, hyperlipidemia, smoking, and much complex coronary anatomy. These factors are closely related to the occurrence and development of atherosclerosis and are associated with the increase in adverse cardiovascular and cerebrovascular events. The high-risk characteristics of patients with prior stroke undergoing PCI call for a better line of treatment strategy from the heart.

\section{Worse Long-Term Prognosis of Patients With Prior Stroke}

Stroke has become the leading cause of death and disabilityadjusted life-years lost in China (16). But for CHD patients with prior stroke who underwent PCI, the prognosis remained controversial. Some studies found that patients with prior stroke usually suffered from more events and higher mortality $(17,18)$. Others showed similar incidence of non-cerebrovascular adverse events between patients with or without prior stroke and that PCI was considered to be safe and effective for CHD patient with prior stroke $(7,19)$.

In this study, although in-hospital outcomes were similar between the two groups, the incidence of adverse cardiovascular and cerebrovascular events of patients with prior stroke was significantly higher during a long-term follow-up of 5 years. This might be due to more risk factors and more multi-vessel disease in patients with prior stroke. In our propensity-matched cohort, however, no difference in 5-year ischemic and hemorrhagic events was found between the two groups, with the exception of recurrent stroke. In general, patients with prior stroke were at a higher risk of cardio-cerebrovascular ischemic and hemorrhagic events, especially recurrent stroke.

\section{Prior Stroke as a Strong Risk Predictor of Future Stroke}

Known for its high prevalence, mortality, and disability, stroke has also been infamous for high rate of recurrence (20). Prior stroke was considered to be a risk factor of recurrent stroke $(21,22)$. In the current study, the multivariable analysis identified prior stroke as an independent risk predictor of future stroke, even after propensity score matching. In addition, this study also shows a significant interaction between ACS, hypertension, diabetes, and prior stroke history, which will significantly increase the risk of future stroke. Hypertension and diabetes history are traditional risk factors of cardiovascular and cerebrovascular diseases. It is suggested that the risk of future stroke in patients combined with prior stroke history and cardiovascular and cerebrovascular risk factors will persist in the long-term follow-up.

\section{Potential Clinical Implications}

This study suggested that it was typical for CHD patients undergoing PCI to have a prior stroke history. These patients had more risk factors of atherosclerosis and more complex vascular condition. Despite standard secondary prevention, patients with prior stroke fared worse in the long run, mainly due to an elevated risk of recurrent stroke. Even after confounder adjustment with both propensity matching and multivariable regression, risk of recurrent stroke in patients with prior stroke remained about two-fold higher than in those without stroke history. Therefore, it is reasonable to suggest that such patients need to receive PCI. A preprocedural consultation involving neurologists and interventional cardiologists might provide a better treatment regimen for additional long-term benefit.

\section{LIMITATION}

Several limitations have to be taken into consideration in this study. First, the data of this study came from a single clinical center, which might not represent the general population. Second, the population of this study is mainly Asian patients, which may be a reason of an expected higher prevalence of intracranial atherosclerosis as a cause of stroke and an evident link with coronary atherosclerosis, but it is challenging to extend to other populations. Third, the prior stroke history was self-reported by patients, and we cannot confirm whether it is hemorrhagic or ischemic. Otherwise, the risk of recall bias would exist. Fourth, the observational design of this study with unmeasured confounders may preclude the definitive conclusion, and additional study may be needed to confirm the observations.

\section{CONCLUSION}

With standard secondary prevention, CHD patients with prior stroke who underwent PCI still had a higher incidence of 5-year long-term adverse cardiovascular and cerebrovascular events, especially stroke. Prior stroke was a strong risk predictor of future stroke events.

\section{DATA AVAILABILITY STATEMENT}

The raw data supporting the conclusions of this article will be made available by the authors, without undue reservation.

\section{ETHICS STATEMENT}

The studies involving human participants were reviewed and approved by Ethical Application No.: IRB2012-BG-006, 
Approval No.: 2013-449. The patients/participants provided their written informed consent to participate in this study.

\section{AUTHOR CONTRIBUTIONS}

J-jX: contributed to project design and article writing. S-dJ, PZ, LJ, PJ, and YS: contributed to data collection and arrangement. $\mathrm{X}-\mathrm{yZ}$, JC, and R-lG: contributed to project design. J-xL: contributed to data sorting and statistics. S-bQ and Y-jY: contributed to project design and operation. BX: project design

\section{REFERENCES}

1. Feigin VL, Forouzanfar MH, Krishnamurthi R, Mensah GA, Connor M, Bennett DA, et al. Global and regional burden of stroke during 1990-2010: findings from the Global Burden of Disease Study 2010. Lancet. (2014) 383:245-54. doi: 10.1016/s0140-6736(13)61953-4

2. Ducrocq G, Amarenco P, Labreuche J, Alberts MJ, Mas JL, Ohman EM, et al. A history of stroke/transient ischemic attack indicates high risks of cardiovascular event and hemorrhagic stroke in patients with coronary artery disease. Circulation. (2013) 127:730-8. doi: 10.1161/CIRCULATIONAHA.112.141572

3. Prosser J, MacGregor L, Lees KR, Diener HC, Hacke W, Davis S, et al. Predictors of early cardiac morbidity and mortality after ischemic stroke. Stroke. (2007) 38:2295-302. doi: 10.1161/STROKEAHA.106.471813

4. Levine GN, Bates ER, Blankenship JC, Bailey SR, Bittl JA, Cercek B, et al. ACCF/AHA/SCAI guideline for percutaneous coronary intervention: a report of the American College of Cardiology Foundation/American Heart Association Task Force on Practice Guidelines and the Society for Cardiovascular Angiography and Interventions. Circulation. (2011) 124:e574651. doi: 10.1161/CIR.0b013e31823ba622

5. Chen Z, Venkat P, Seyfried D, Chopp M, Yan T, Chen J. Brain-heart interaction: cardiac complications after stroke. Circ Res. (2017) 121:451-68. doi: 10.1161/CIRCRESAHA.117.311170

6. Olubowale OT, Safford MM, Brown TM, Durant RW, Howard VJ, Gamboa $\mathrm{C}$, et al. Comparison of expert adjudicated coronary heart disease and cardiovascular disease mortality with the national death index: results from the REasons for Geographic And Racial Differences in Stroke (REGARDS) Study. J Am Heart Assoc. (2017) 6:e004966. doi: 10.1161/JAHA.116.004966

7. Xu JJ, Song Y, Jiang P, Gao Z, Li JX, Chen J, et al. Effect of prior stroke on longterm outcomes of percutaneous coronary interventions in Chinese patients: a large single-center study. Catheter Cardiovasc Interv. (2019) 93:E75-80. doi: $10.1002 /$ ccd.27705

8. Thygesen K, Alpert JS, Jaffe AS, Simoons ML, Chaitman BR, White HD, et al. Third universal definition of myocardial infarction. Glob Heart. (2012) 7:275-95. doi: 10.1016/j.gheart.2012.08.001

9. Cutlip DE, Windecker S, Mehran R, Boam A, Cohen DJ, van Es GA, et al. Clinical end points in coronary stent trials: a case for standardized definitions. Circulation. (2007) 115:2344-51. doi: 10.1161/CIRCULATIONAHA.106.685313

10. Mehran R, Rao SV, Bhatt DL, Gibson CM, Caixeta A, Eikelboom J, et al. Standardized bleeding definitions for cardiovascular clinical trials: a consensus report from the Bleeding Academic Research Consortium. Circulation. (2011) 123:2736-47. doi: 10.1161/CIRCULATIONAHA.110.009449

11. Yadav M, Palmerini T, Caixeta A, Madhavan MV, Sanidas E, Kirtane AJ, et al. Prediction of coronary risk by SYNTAX and derived scores: synergy between percutaneous coronary intervention with taxus and cardiac surgery. J Am Coll Cardiol. (2013) 62:1219-30. doi: 10.1016/j.jacc.2013.06.047

12. Venketasubramanian N, Yoon BW, Pandian J, Navarro JC. Stroke epidemiology in South, East, and South-East Asia: a review. J Stroke. (2017) 19:286-94. doi: 10.5853/jos.2017.00234

13. Colantonio LD, Gamboa CM, Kleindorfer DO, Carson AP, Howard VJ, Muntner P, et al. Stroke symptoms and risk for incident coronary heart disease in the REasons for Geographic And Racial Differences in Stroke (REGARDS) study. Int J Cardiol. (2016) 220:122-8. doi: 10.1016/j.ijcard.2016.06.030 and overall plan. All authors contributed to the article and approved the submitted version.

\section{ACKNOWLEDGMENTS}

We would like to thank all individuals who participated in this study for their contribution to data acquisition, patient selection, and quality control. We are grateful to the staff in the Department of Cardiology and Catheterization Laboratory, Fuwai Hospital, for their research contributions.

14. Gruneir A, Griffith LE, Fisher K, Panjwani D, Gandhi S, Sheng $\mathrm{L}$, et al. Increasing comorbidity and health services utilization in older adults with prior stroke. Neurology. (2016) 87:2091-8. doi: 10.1212/WNL.0000000000003329

15. Khafaji HAR, Sulaiman K, Singh R, AlHabib KF, Asaad N, Alsheikh-Ali A, et al. Clinical characteristics, precipitating factors, management and outcome of patients with prior stroke hospitalised with heart failure: an observational report from the Middle East. BMJ Open. (2015) 5:e007148. doi: 10.1136/bmjopen-2014-007148

16. Wu S, Wu B, Liu M, Chen Z, Wang W, Anderson CS, et al. Stroke in China: advances and challenges in epidemiology, prevention, and management. Lancet Neurol. (2019) 18:394-405. doi: 10.1016/S1474-4422(18)3 0500-3

17. Li YJ, Rha SW, Chen KY, Jin Z, Minami Y, Wang L, et al. Clinical characteristics and mid-term outcomes of acute myocardial infarction patients with prior cerebrovascular disease in an Asian population: lessons from the Korea Acute Myocardial Infarction Registry. Clin Exp Pharmacol Physiol. (2010) 37:581-6. doi: 10.1111/j.1440-1681.2010.05363.x

18. Fonarow GC, Saver JL, Smith EE, Broderick JP, Kleindorfer DO, Sacco RL, et al. Relationship of national institutes of health stroke scale to 30-day mortality in medicare beneficiaries with acute ischemic stroke. Am Heart Assoc. (2012) 1:42-50. doi: 10.1161/JAHA.111.000034

19. Natsuaki M, Morimoto T, Watanabe H, Nakagawa Y, Furukawa Y, Kadota K, et al. Ischemic and bleeding risk after percutaneous coronary intervention in patients with prior ischemic and hemorrhagic stroke. J Am Heart Assoc. (2019) 8:e013356. doi: 10.1161/JAHA.119.013356

20. Sharma M, Hart RG, Connolly SJ, Bosch J, Shestakovska O, Ng KKH, et al. Stroke outcomes in the COMPASS trial. Circulation. (2019) 139:1134-45. doi: 10.1161/CIRCULATIONAHA.118.035864

21. Quinn CT. Breakthrough: new guidance for silent cerebral ischemia and infarction in sickle cell disease. Hematology Am Soc Hematol Educ Program. (2014) 2014:438-43. doi: 10.1182/asheducation-2014.1.438

22. Calvet D, Song D, Yoo J, Turc G, Sablayrolles JL, Choi BW, et al. Predicting asymptomatic coronary artery disease in patients with ischemic stroke and transient ischemic attack: the PRECORIS score. Stroke. (2014) 45:82-6. doi: 10.1161/STROKEAHA.113.003414

Conflict of Interest: The authors declare that the research was conducted in the absence of any commercial or financial relationships that could be construed as a potential conflict of interest.

Publisher's Note: All claims expressed in this article are solely those of the authors and do not necessarily represent those of their affiliated organizations, or those of the publisher, the editors and the reviewers. Any product that may be evaluated in this article, or claim that may be made by its manufacturer, is not guaranteed or endorsed by the publisher.

Copyright (c) $2021 \mathrm{Xu}$, Jia, Zhu, Jiang, Jiang, Song, Zhao, Li, Chen, Yang, Gao, Qiao, Xu and Yuan. This is an open-access article distributed under the terms of the Creative Commons Attribution License (CC BY). The use, distribution or reproduction in other forums is permitted, provided the original author(s) and the copyright owner(s) are credited and that the original publication in this journal is cited, in accordance with accepted academic practice. No use, distribution or reproduction is permitted which does not comply with these terms. 